\title{
Measurement of mixed-mode fracture parameters near cracks in homogeneous and bimaterial beams
}

\author{
HAREESH V. TIPPUR and SREEGANESH RAMASWAMY \\ Department of Mechanical Engineering, Auburn University, Auburn, Alabama 36849, USA
}

Received 4 September 1992; accepted in revised form 27 March 1993

\begin{abstract}
An investigation of deformation fields and evaluation of fracture parameters near mixed-mode cracks in homogeneous and bimaterial specimens under elastostatic conditions is undertaken. A modified edge notched flexural geometry is proposed for testing bimaterial interface fracture toughness. The ability of the specimen in providing a fairly wide range of mode mixities is demonstrated through direct optical measurements and a simple fiexural analysis. A full field optical shearing interferometry called 'Coherent Gradient Sensing' (CGS) is used to map crack tip deformations in real time. Experimental measurements and predictions based on beam theory are found to be in good agreement. Also, for a large stiffness mismatch bimaterial system, the interface crack initiation toughness is evaluated as a function of the crack tip mode mixity.
\end{abstract}

\section{Introduction}

One of the primary causes of composite material failure is crack initiation and crack growth along weak/brittle interfaces during service. Interface fracture mechanics is quite distinct from homogeneous counterparts and alternative approaches are used in understanding the phenomenon. Across an interface, there exists a stiffness mismatch due to which the crack tip fields and hence the failure processes are intrinsically mixed-mode even when the remote loading is pure tension or pure shear. Consequently, interface fracture toughness is dependent on mode-mixity. Bimaterial interface fracture mechanics research goes back to the early works of Williams [1]. It led to the observation of an oscillatory stress singularity of the form $r^{-1 / 2+i \varepsilon}$, where $r, \varepsilon$ and $i$ are radial distance from the crack tip, the stiffness mismatch parameter, and $\sqrt{-1}$, respectively. Rice and Sih [2] and Sih and Rice [3] provided explicit relations for 2-D near tip stresses and related them to remote elastic stress fields. Erdogan [4] and England [5] have solved various 2-D models for single and multiple crack geometries. These solutions imply crack flank interpenetration at small distances behind the crack tip. This contradicts the assumption of traction free crack surfaces. Comninou [6] suggested a model with a frictionless contact between the crack surfaces which eliminated crack flank interpenetration of the crack faces. However, this formulation led to some unusual crack tip features namely, spreading of an interface crack under tension is intimately connected with failure in shear. Knowles and Sternberg [7] have shown that the oscillatory behavior can be eliminated through the incorporation of material nonlinearity. Shih and Asaro [8], in their two-dimensional, nonlinear finite element computation, have also observed this. Sharma and Aravas [9] have reported studies on an interface crack between an elastoplastic material and a rigid substrate. They have studied the regions of dominance of different solutions. In view of these recent developments, Rice [10] has suggested that a 'small scale contact approach' is appropriate from an engineering point of view. Hutchinson [11] has enunciated the concept of a complex stress intensity factor $K\left(=K_{1}+i K_{2}\right)$ for bimaterial crack tip fields. Recently, O'Dowd et al. [12] have presented 
finite element analysis of various test geometries for the purpose of measuring interfacial toughness. Hutchinson and Suo [13] have summarized the fracture mechanics research of layered materials to date in a recent review article.

Experimental investigations on interface fracture include those of Liechti and Knauss [14] who interferometrically studied the debonding of a sandwiched elastomer in a uniformly loaded strip in which extensive three-dimensional deformations are observed. Later studies by Liechti and Chai [15] have used hybrid interferometric measurements and finite element calculations for crack opening displacement and hence crack tip parameter measurements under remote bond normal and bond tangential displacements. Crack tip field measurements in a bimaterial model made out of two photoelastic materials have been reported by Chiang et al. [16]. Cao and Evans [17] and Charalambides et al. [18] have performed experimental and numerical studies to infer crack tip parameters. The latter work proposes a four point bend geometry for interface fracture testing. Tippur and Rosakis [19] have reported experimental investigations on bimaterial systems consisting of PMMA and Aluminum for quasi-static and dynamic cases through direct optical measurements and have observed unusually high crack velocities during dynamic loading.

The primary objectives of the present investigation are to (i) optically map crack tip deformations and then measure fracture parameters in homogeneous and bimaterial beam specimens under remote mixed-mode loading, and (ii) develop a practical homogeneous and bimaterial fracture specimen, that provides a relatively wide range of mode mixities and that involves a simple and compact loading configuration. .A single edge crack geometry is preferred in order to achieve greater consistency in the experimental results by reducing symmetry requirements that are present in other bimaterial test specimens with two crack tips $[12,18]$. A flexural specimen also provides a compact and easy-to-load test configuration. In this investigation, crack tip deformations are mapped directly using the full field, optical method of transmission coherent gradient sensing. A flexural analysis of the geometry is carried out to provide theoretical comparisons for the experimental results. In what follows, the ability of the specimen to provide a relatively wide range of mode mixities is first demonstrated on homogeneous specimens and then its use extended to interface fracture toughness testing.

\section{Coherent gradient sensing (CGS)}

CGS is a wave front shearing interferometry that provides gradients of deformations near cracks [20]. It gives full field information in real time and the sensitivity of measurement is easily controllable. The method is relatively insensitive to random vibrations and rigid motions. Also, it can be used with both opaque (reflection mode) as well as transparent solids (transmission mode).

The schematic of transmission CGS set up is shown in Fig. 1. Figure 2 shows the photograph of the actual experimental set up. A collimated laser beam is transmitted through a transparent fracture specimen. In the vicinity of a deformed crack, non-uniform stress fields exist. Hence the incident planar wavefront gets perturbed upon propagation through the crack tip region. The object wave front can be viewed to be made of several locally planar wave fronts with propagation vectors oriented in different directions. If $\beta_{1}\left(x_{1}, x_{2}\right), \beta_{2}\left(x_{1}, x_{2}\right)$ and $\beta_{3}\left(x_{1}, x_{2}\right)$ 


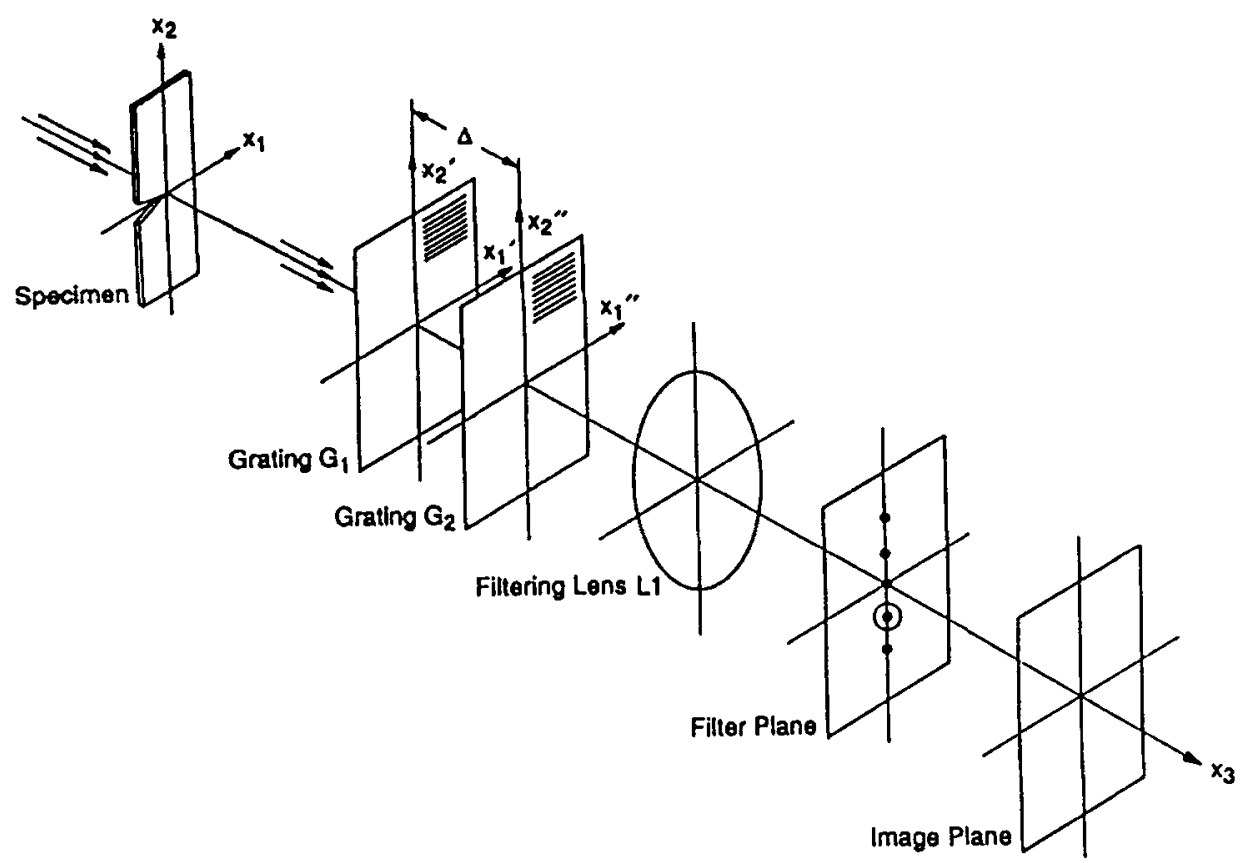

Fig. 1. Schematic of the experimental set up of transmission CGS.

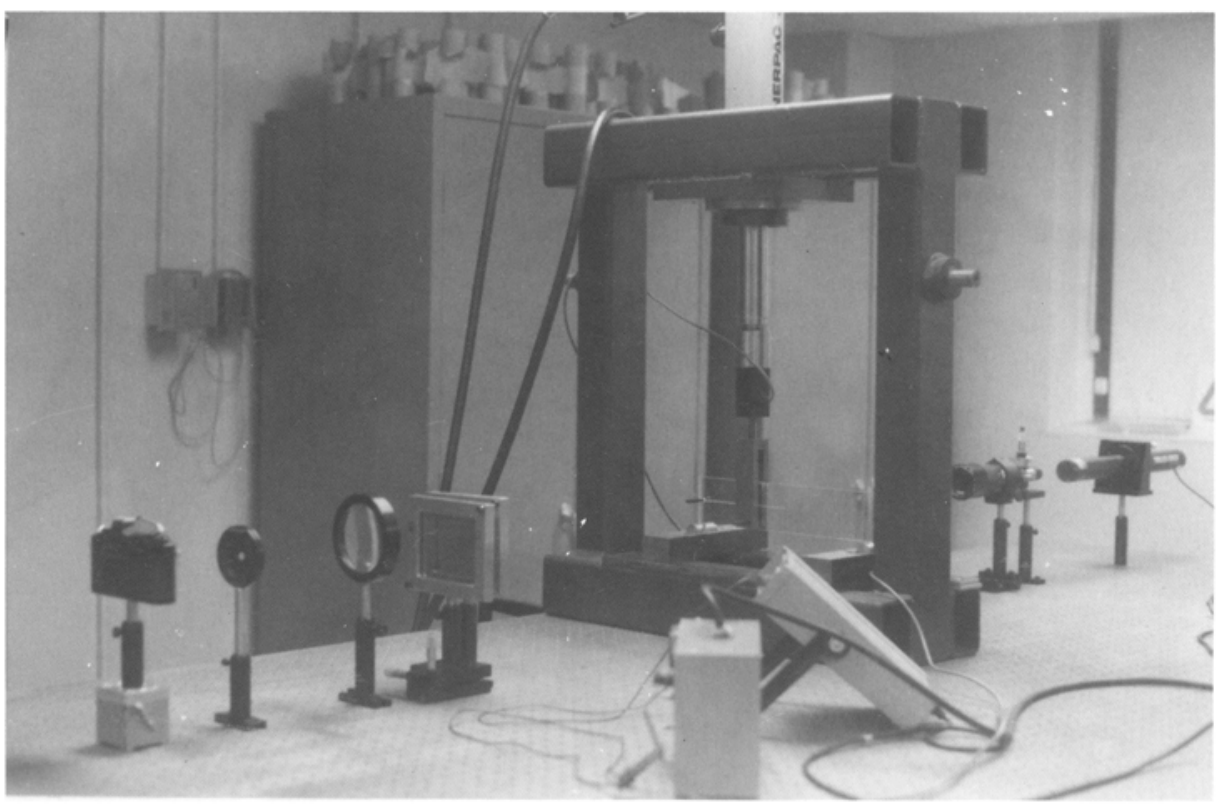

Fig. 2. Photograph of the experimental set up.

denote the direction cosines of the local propagation vector $\mathbf{d}$, then we can write

$$
\mathbf{d}=\beta_{i} \mathbf{e}_{i}, \quad i=1,2,3,
$$

where $\mathbf{e}_{i}$ represent unit normals along Cartesian coordinates. The object wave front, which 


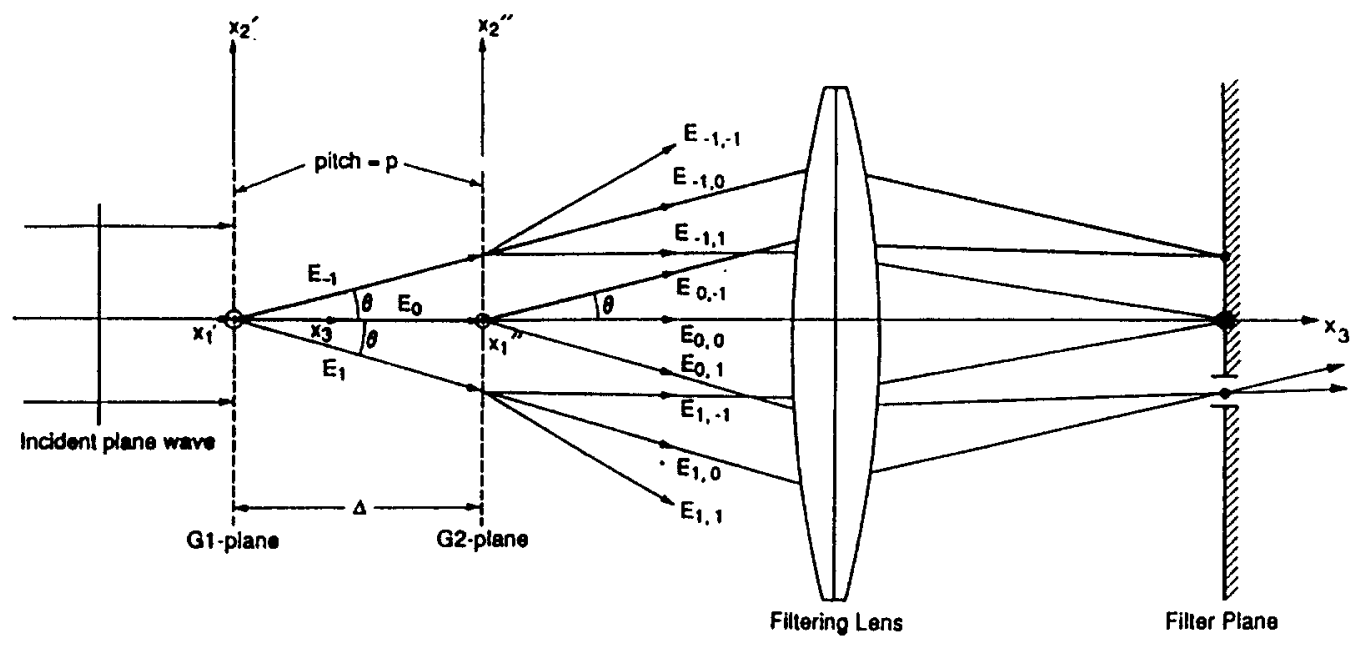

Fig. 3. Working principle of CGS.

carries information of the crack tip deformations, subsequently undergoes a series of diffractions as it propagates through two identical high density Ronchi gratings $G_{1}$ and $G_{2}$ (grating pitch $p$ ) which are spatially separated by a distance $\Delta$ along the optical axis (Fig. 3). The gratings are chromium-on-glass master gratings with antireflection coatings and have nearly square wave transmission profile. From the schematic shown in Fig. 3, it is clear that the diffracted wave fronts $E_{(0,1)}$ and $E_{(1,0)}$ are spatially sheared versions of the object wave front. The path difference between these two wave fronts is a function of the local direction cosines of the object wave front. Also, because the propagation directions of $E_{(0,1)}$ and $E_{(1,0)}$ are the same, they will come to focus at a common point on the back focal plane of the filtering lens. The spatial frequency content of the object wave front is filtered at the filtering plane and the image is photographed.

Through a first order diffraction analysis, Tippur et al. [20] have related the interference patterns to the direction cosines, $\beta_{1}$ and $\beta_{2}$ of the object wave front by the simple relationship,

$$
\beta_{\alpha}=\frac{n_{\alpha} p}{\Delta}, \quad \alpha=1,2 ; \quad n_{\alpha}=0, \pm 1, \pm 2, \ldots,
$$

where $n_{\alpha}$ represents fringe orders. The above relationship is valid for small angular deflections of the light rays $\left(\beta_{3} \approx 1\right)$. The direction cosines can be further related to the deformation field under plane stress assumptions. A detailed analysis has shown that, for transmission CGS, the following governing relations between mechanical fields and optical patterns exist

$$
\beta_{\alpha}=c B \frac{\partial\left(\sigma_{11}+\sigma_{22}\right)}{\partial x_{\alpha}}=\frac{n_{\alpha} p}{\Delta}, \quad \alpha=1,2
$$

where $\sigma_{11}, \sigma_{22}$ are the through-the-thickness average of normal stress components, $c$ is the elasto-optical constant for the material and $B$ is the undeformed plate thickness. 


\section{Mixed-mode deformation in homogeneous specimens}

First, the feasibility of the beam specimen for mixed-mode fracture studies is demonstrated through crack tip measurements in homogeneous specimens.

\subsection{Test specimens and optical measurements}

The specimen geometry and the loading configuration used are shown in Fig. 4. Test specimens are made from commercially available PMMA sheets of nominal thickness $9 \mathrm{~mm}$ (manufactured by CYRO Industries, Mt. Arlington, NJ). A $0.5 \mathrm{~mm}$ thick band saw is used to cut transverse slits of different lengths in these fracture specimens. Sufficient care is exercised during cutting to minimize residual stresses along the crack flanks and at the crack tip. The cracked edge of the specimen is further notched to a depth of $12.5 \mathrm{~mm}$ to produce a slot of width of $6.25 \mathrm{~mm}$. A pin of $6.25 \mathrm{~mm}$ diameter is housed in this slot during the test. Upon loading, the pin induces a reactive load between the upper and lower arms of the beam. A loose hole of $6.25 \mathrm{~mm}$ diameter in the lower arm has been used for applying the load $P$ shown in Fig. 4. The height of the beam $H=75 \mathrm{~mm}$ and the span is $L=330 \mathrm{~mm}$. Six different crack length $(a)$ to loading distance $(l)$ ratios, namely $(a / l)=1.39,1.65,1.91,2.15,2.41$ and 2.51 , are studied.

A photograph of the experimental set-up is shown in Fig. 2. The specimen is loaded by a hydraulically operated plunger. A $0-3000 \mathrm{lb}$, universal load cell is used for measuring the applied load $P$. The load cell is included in the set-up in such a way that one end of it is attached to the plunger while its other end is connected to a loading fork. The fork forms a pin joint with the specimen and applies the load $P$ to the specimen.

Transmission CGS has been used in the present investigation. A collimated laser beam of diameter $50 \mathrm{~mm}$ is centered around the crack tip and transmitted through the specimens in these experiments. The object wave front shearing is accomplished by two chromium-on-glass master line gratings of pitch, $p=0.025 \mathrm{~mm}$ and the separation distance between gratings, $\Delta$ is $39 \mathrm{~mm}$. A series of discrete diffraction spots are visible on the back focal plane of the filtering lens. Either the +1 or -1 diffraction orders is filtered out and the resulting interference patterns are photographed at the image plane. Note that the imaging system, consisting of the filtering lens and the camera back, is focussed on the object plane. Typical fringe patterns around the crack tip for two $(a / l)$ ratios when the grating lines are perpendicular to the $x_{1}$-axis, are shown in

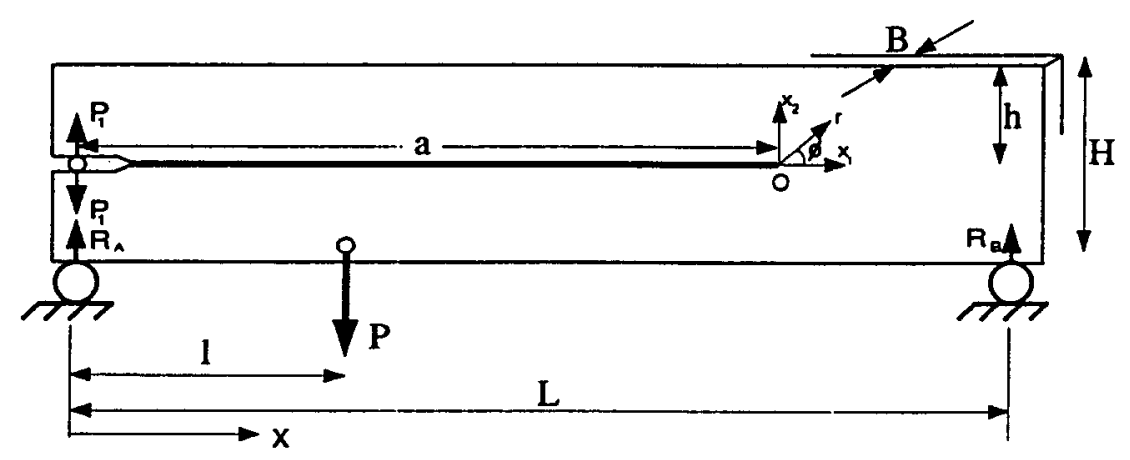

Fig. 4. Transversely cracked homogeneous specimen. 
Figs. 5(a), (b). In regions around the crack tip, where plane stress is a good approximation, these fringes represent contours of $c B\left(\partial\left(\sigma_{11}+\sigma_{22}\right) / \partial x_{1}\right)$, where $\sigma_{11}$ and $\sigma_{22}$ are the thickness averages of normal stress components. The sensitivity of measurement is $6.4 \times 10^{-4}$ radians per fringe and the elasto-optic constant from the model material is $-0.9 \times 10^{-4} \mathrm{~m}^{2} / \mathrm{N}$.

\subsection{Crack tip fields}

The method of transmission CGS provides gradients of $\left(\sigma_{11}+\sigma_{22}\right)$ with respect to the $x_{1}$ - or $x_{2}$-coordinate. The measurements performed in this work are restricted to $x_{1}$-gradients only. Following Williams [21], for a semi-infinite mixed-mode elastic crack, we can write

$$
\frac{\partial\left(\sigma_{11}+\sigma_{22}\right)}{\partial x_{1}}=\sum_{N=1}^{\infty}\left(\frac{1}{2} N-1\right) r^{((N / 2)-2)}\left[A_{N} \cos \left(\frac{1}{2} N-2\right) \phi+B_{N} \sin \left(\frac{1}{2} N-2\right) \phi\right]
$$

where the coefficients $A_{N}$ and $B_{N}$ are the undetermined constants of the series. Here, $A_{1}$ and $B_{1}$ are proportional to the mode-I and mode-II stress intensity factors $K_{1}$ and $K_{\mathrm{II}}$ respectively, and $A_{2}, \ldots, A_{N}, B_{2}, \ldots, B_{N}$ are the constant coefficients of higher order terms. Combining (3) and (4),

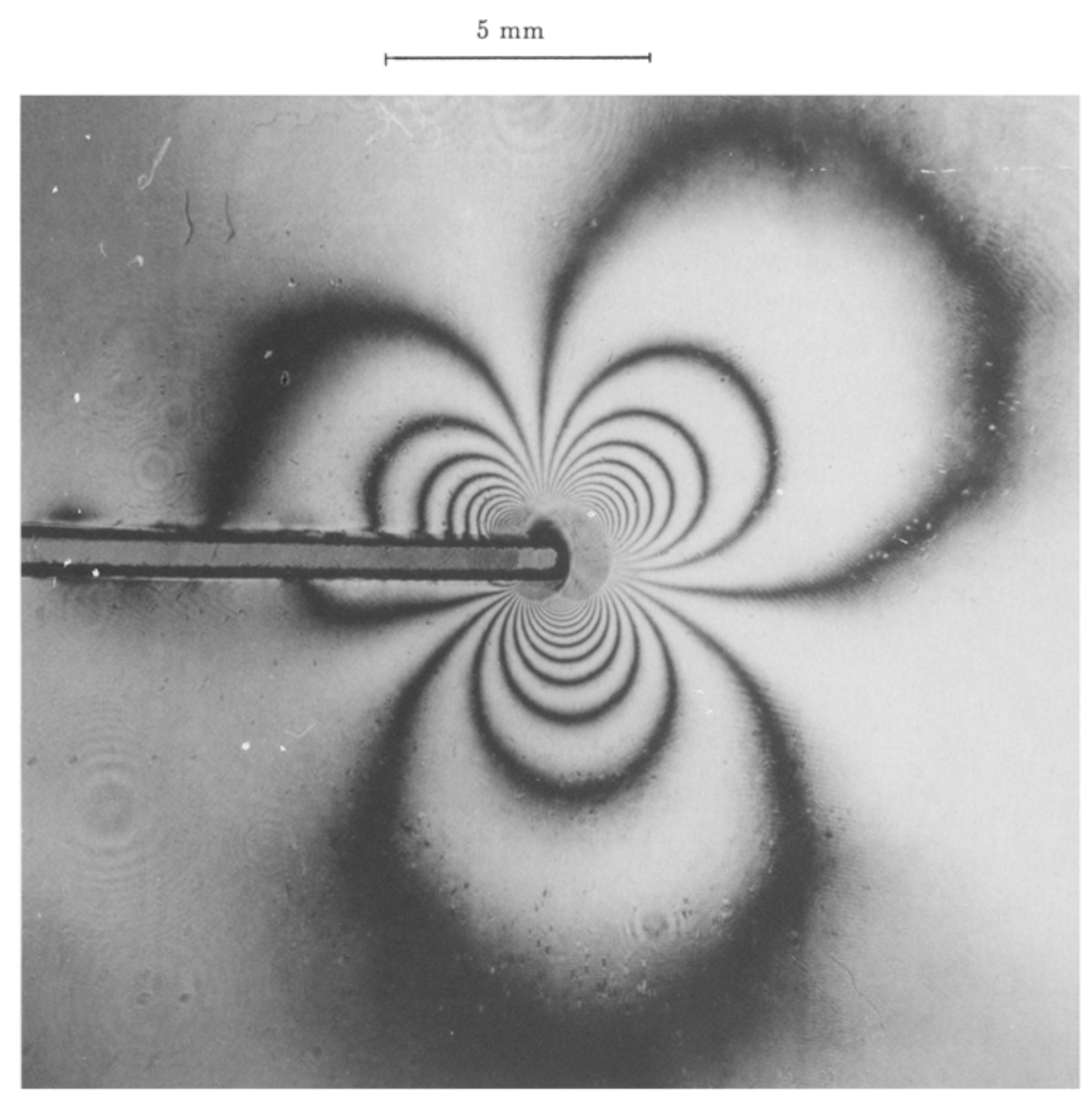

Fig. 5(a). Transmission CGS fringes representing contours of $\partial\left(\sigma_{11}+\sigma_{22}\right) / \hat{c} x_{1}$ for the specimen with $(a / l)=1.39$. 


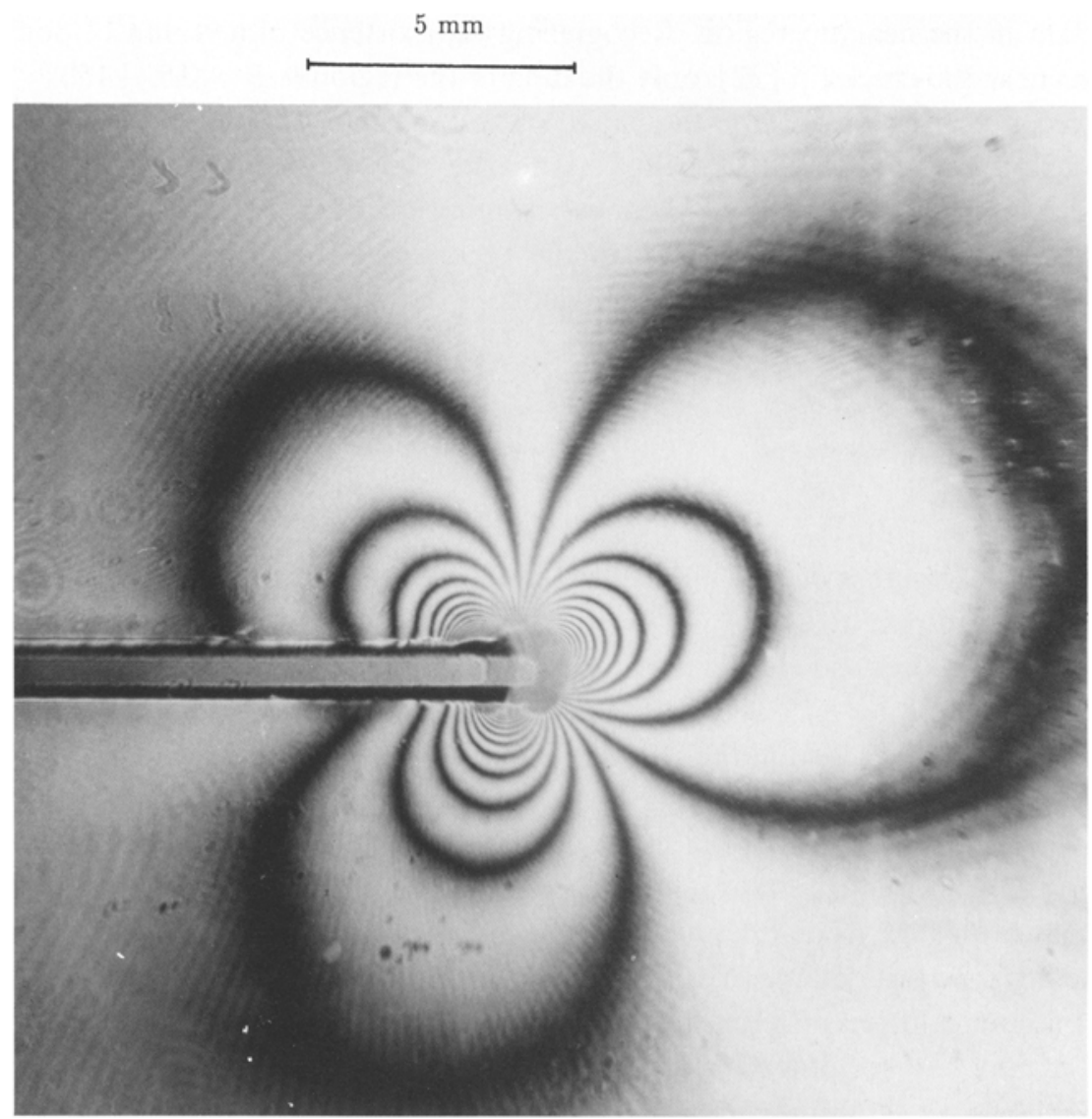

Fig. 5(b). Transmission CGS fringes representing contours of $\partial\left(\sigma_{11}+\sigma_{22}\right) /\left(\partial x_{1}\right.$ for the specimen with $(a / l)=2.41$.

deformation fields can be related to the CGS interference patterns

$$
\begin{aligned}
c B \frac{\partial\left(\sigma_{11}+\sigma_{22}\right)}{\partial x_{1}} & =c B \sum_{N=1}^{\infty}\left(\frac{1}{2} N-1\right) r^{((N / 2)-2)}\left[A_{N} \cos \left(\frac{1}{2} N-2\right) \phi+B_{N} \sin \left(\frac{1}{2} N-2\right) \phi\right], \\
& =\frac{n_{1} p}{\Delta}
\end{aligned}
$$

where $n_{1}(=0, \pm 1, \pm 2 \ldots)$ represent the fringe orders. Now we shall define a $K$-dominant field as one in which the contribution from the higher order terms is negligible when compared to the first term $(N=1)$. Thus, for $K$-dominance, $(5)$ reduces to,

$$
\frac{c B r^{-3 / 2}}{\sqrt{2 \pi}}\left[-K_{\mathrm{I}} \cos (3 \phi / 2)+K_{\mathrm{II}} \sin (3 \phi / 2)\right]=\frac{n_{1} p}{\Delta} .
$$

\subsection{Measurement of $K_{\mathrm{I}}$ and $K_{\mathrm{II}}$ from interference patterns}

To extract stress intensity factors from the fringe patterns, we use a multi-parameter leastsquares data analysis. The fringe patterns are digitized to obtain fringe location $(r, \phi)$ and fringe 
order $\left(n_{1}\right)$ data in the near tip region. Recognizing the existence of a region of dominant 3-D deformations near the crack tip [22], only the data in the region $\left(r / B \geqslant 0.5,-150^{\circ} \leqslant \phi \leqslant 150^{\circ}\right)$ near the crack tip is used in the analysis. At distances beyond $(r / B)=0.5$, nonsingular contributions to the stress field can not be generaliy ignored owing to the finite size of the specimen. Thus, $K$-dominance assumptions are relaxed and higher order terms (in (5)) are included in the analysis of the experimental data. Denoting the right hand sides of (5) by $Y$ and $F$, respectively, we define a function $T\left(A_{1}, A_{2}, \ldots, A_{N}, B_{1}, B_{2}, \ldots\right.$, $\left.B_{N} ; r, \phi\right)$ as

$$
\Phi=\sum_{i=1}^{M}\left[Y_{i}-F_{i}\right]^{2}
$$

where $M$ is the total number of data points used in the analysis. In the curve fitting procedure, $\Phi$ is minimized with respect to $A_{1}, \ldots, A_{N}, B_{1}, \ldots, B_{N}$. The values of $\sqrt{(\pi / 2)} A_{1}$ and $\sqrt{(\pi / 2)} B_{1}$ corresponding to the least squares data fit are the experimentally determined stress intensity factors and are denoted by $K_{\mathrm{I}}^{\exp }$ and $K_{\mathrm{II}}^{\exp }$.

The size of the specimen used in the study being finite, the influence of higher order terms can not be generally ignored. Inclusion of higher order terms in the data analysis is seen to improve the agreement between the least-squares fit and the optical data. However, the inclusion of higher order terms beyond a certain number tends to produce larger disagreement between the fit and the data. This may be attributed to the finite number of data points and the inherent 'noise' in the digitized data due to errors associated with locating fringe centers. However, there exists an optimum number of terms in the expansion which will provide the 'best' fit to the digitized data. Any number of terms different from this optimum number would produce larger deviations between the fit and the data. In order to determine the appropriate number of terms to be used in interpreting the fringes, the Standard Deviation between the radial locations of the fit and the data points along discrete directions is used (see [23]) for details). An example of the least squares fit for an $(a / l)$ ratio of 1.91 are shown in Figs. 6(a), (b).

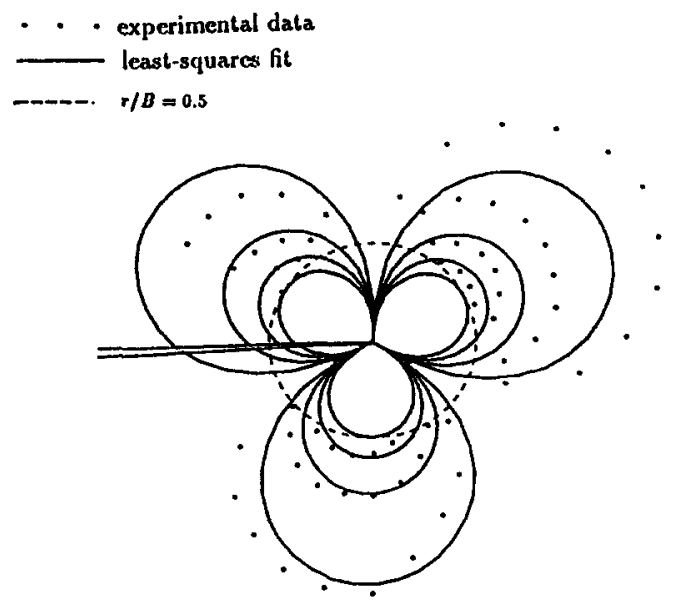

Fig. $\sigma(a)$. Least-squares fit with $K$-dominant terms $(N=1)$ and data points for an $(a / l)=1.91$.

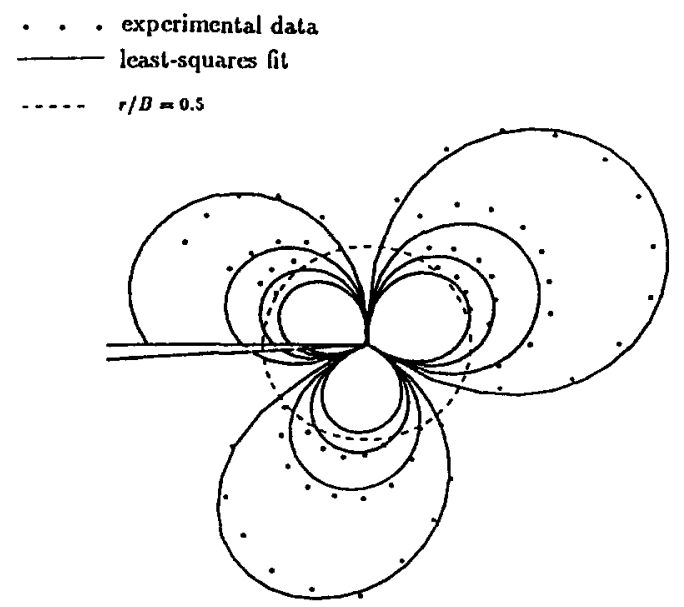

Fig. $6(b)$. Least-squares fit with higher order terms ("best fit'; $N=5)$ and data points for $(a / L)=1.91$. 


\subsection{Beam analysis}

Using flexural analysis, for an applied load $P$ acting on the beam (Fig. 4), the energy release rate $G^{b t}$ can be expressed as $[23,13]$

$$
G^{b t}=\frac{1}{2 B}\left[\frac{M_{1}^{2}}{E I_{1}}+\frac{M_{2}^{2}}{E I_{2}}-\frac{\left(M_{1}+M_{2}\right)^{2}}{E I_{0}}\right],
$$

where $B$ is the thickness of the specimen, $I_{1}=\frac{1}{12} B h^{3}, I_{2}=\frac{1}{12} B(H-h)^{3}$ and $I_{0}=\frac{1}{12} B H^{3}$ are the moments of inertia of the cross-sections of the upper arm, the lower arm and the uncracked portion respectively, $E$ is the Young's modulus of the material, and,

$$
\begin{aligned}
& M_{1}=P_{1} a, \\
& M_{2}=\left(R_{A}-P_{1}\right) a-P(a-l),
\end{aligned}
$$

where $R_{A}[=P(1-(l / L))]$ is the left support reaction. Here,

$$
P_{1}=\frac{P}{2}\left[\frac{3}{2}\left(\frac{l}{a}\right)-\frac{1}{2}\left(\frac{l}{a}\right)^{3}-\left(\frac{l}{L}\right)\right],
$$

represents the reactive force between the upper and the lower arms of the cracked beam. It should be mentioned that the shear contribution to the energy release rate calculation is not accounted for in (8). However, it is shown that the shear contribution is negligible in the range of crack lengths considered [23].

In order to calculate stress intensity factors based on beam theory $K_{I}^{b t}$ and $K_{\mathrm{II}}^{b t}$ from $G^{b t}$, a mode partitioning method outlined by Williams [24], is used. It should be pointed out, however, that the method is applicable only to the special case of $\xi=h / H=0.5$ [23]. The mode partitioning method is basically a moment decomposition technique in which the system of moments at the crack tip $M_{1}$ and $M_{2}$ can be written in terms of $M_{\mathrm{I}}$ and $M_{\mathrm{II}}$, where $M_{\mathrm{I}}$ and $M_{\mathrm{II}}$, respectively, represent the moments responsible for pure mode-I and pure mode-II deformations at the crack tip. This moment decomposition is based on the assumption that the radii of curvature of the upper and lower beams should be equal in magnitude when the crack propagates under pure mode-I or pure mode-II. For pure mode-I deformation, the curvatures of the upper and lower beams have opposite signs, while they are identical for pure mode-II deformation, as shown schematically in Fig. 7. Thus it follows that for $\xi=0.5, M_{1}=M_{1}+M_{\mathrm{II}}$ and $M_{2}=M_{\mathrm{II}}-M_{\mathrm{I}}$. After substituting for $M_{1}$ and $M_{2}$ in (8), and grouping the terms involving $M_{1}$ and $M_{11}$, energy release rate can be expressed as

$$
G^{b t}=\frac{1}{B E I}\left(M_{1}^{2}+\frac{3}{4} M_{\mathrm{II}}^{2}\right)
$$




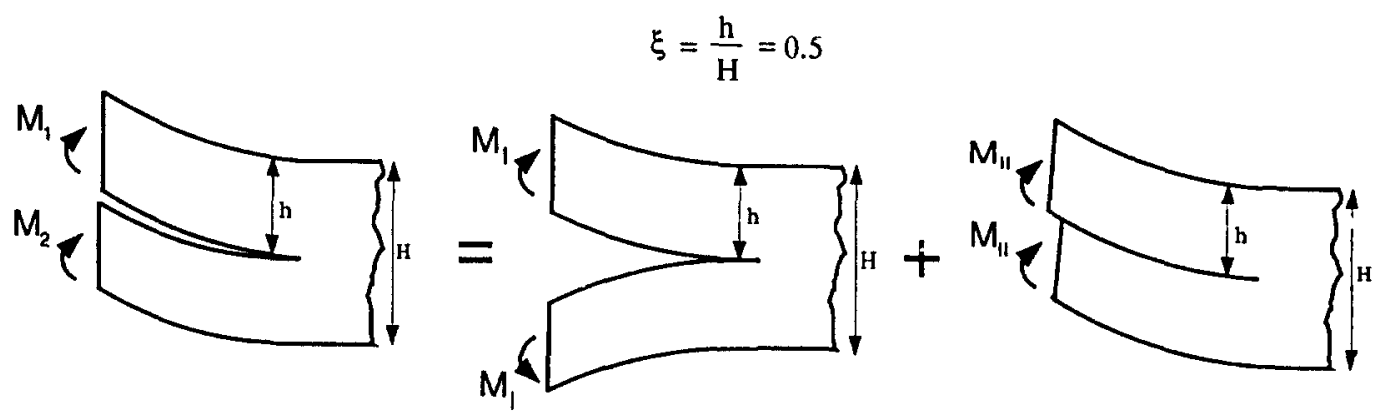

Fig. 7. Mode partitioning by moment decomposition.

Thus, the expression for energy release rate in terms of the energy release rates associated with symmetric deformations $\left(G_{\mathrm{I}}\left(M_{\mathrm{I}}\right)\right)$ and anti-symmetric deformations $\left(G_{\mathrm{II}}\left(M_{\mathrm{II}}\right)\right)$ are as follows

$$
\begin{aligned}
& G_{\mathrm{I}}^{b t}=\frac{\left(K_{\mathrm{I}}^{b t}\right)^{2}}{E}=\left(\frac{3 P^{2} l^{2}}{4 B^{2} E h^{3}}\right)\left[1-\left(\frac{l}{a}\right)^{2}\right]^{2}, \\
& G_{\mathrm{II}}^{b t}=\frac{\left(K_{\mathrm{II}}^{b t}\right)^{2}}{E}=\left(\frac{9 P^{2} l^{2}}{4 B^{2} E h^{3}}\right)\left[1-\left(\frac{a}{L}\right)\right]^{2} .
\end{aligned}
$$

From the above equations, $K_{\mathrm{I}}^{b t}$ and $K_{\mathrm{II}}^{b t}$ can be determined.

\subsection{Results for homogeneous specimens}

The results from the experiments and the theory for different $(a / l)$ ratios are summarized in Figs. 8,9 and Table 1. Figure 8 shows the variations of normalized experimental measurements and beam theory predictions of $K_{\mathrm{I}}$ and $K_{\mathrm{II}}$ with respect to $(a / l)$. The quantity $\sqrt{K_{\mathrm{I}}^{2}+K_{11}^{2}}$ is chosen as the normalization parameter. As noted earlier the mode-mixity varies with $(a / l)$; while $K_{\mathbf{I}}$ increases with $(a / l), K_{\mathrm{II}}$ decreases. The solid lines in Fig. 8 represent beam theory prediction; while the triangles and boxes, represent the experimental results. The measured values shown correspond to the 'best' least-squares fit and they are in good agreement with the beam model. Figure 9 shows a plot of the mode mixity parameter, $\psi\left(=\tan ^{-1} K_{\mathrm{II}} / K_{\mathrm{I}}\right)$ vs. $(a / l)$. Again, the solid line represents prediction based on beam theory and the circles are the experimental measure-

\begin{tabular}{|c|c|c|c|c|c|c|c|c|c|c|c|}
\hline$a / l$ & $P$ & $K_{1}^{b t}$ & $K_{\text {II }}^{b t}$ & $K_{I}^{\exp _{p}}$ & $K_{\mathrm{ll}}^{\exp }$ & $\mathrm{N}$ & $K_{1}^{\exp (b)}$ & $K_{\mathrm{j} 1}^{\exp (b)}$ & $-\psi^{b t}$ & $-\psi^{\text {exp }}$ & $-\psi^{\exp (b)}$ \\
\hline 1.39 & 786 & 0.48 & -1.02 & 0.66 & -1.30 & 4 & 0.60 & -1.14 & 64.8 & 63.1 & 62.2 \\
\hline 1.65 & 723 & 0.64 & -0.89 & 0.84 & -1.01 & 5 & 0.74 & -0.87 & 54.3 & 50.3 & 49.6 \\
\hline 1.91 & 723 & 0.72 & -0.74 & 1.03 & -0.89 & 5 & 0.85 & -0.79 & 45.8 & 40.8 & 42.9 \\
\hline 2.15 & 723 & 0.78 & -0.60 & 1.02 & -0.74 & 5 & 0.83 & -0.64 & 37.6 & 36.0 & 37.6 \\
\hline 2.41 & 736 & 0.84 & -0.48 & 0.97 & -0.67 & 5 & 0.86 & -0.49 & 29.7 & 34.6 & 29.7 \\
\hline 2.51 & 1364 & 1.18 & -0.59 & 1.16 & -0.56 & 5 & 1.02 & -0.47 & 26.6 & 25.8 & 24.7 \\
\hline
\end{tabular}

Table 1. Summary of mixed-mode crack tip measurements from homogeneous beams

$(*)^{\exp }$ and $(*)^{\exp (b)}$ correspond to the $K$-dominant field and the asymptotic field cases, respectively, of the experimental results. $(*)^{b t}$ are the values from beam analysis. SIF values are in $\mathrm{MPa} \sqrt{\mathrm{m}}, \psi$ values are in degrees, and $P$ is in Newtons, $N$ corresponds to the optimum value that produces the best least-squares fit. 


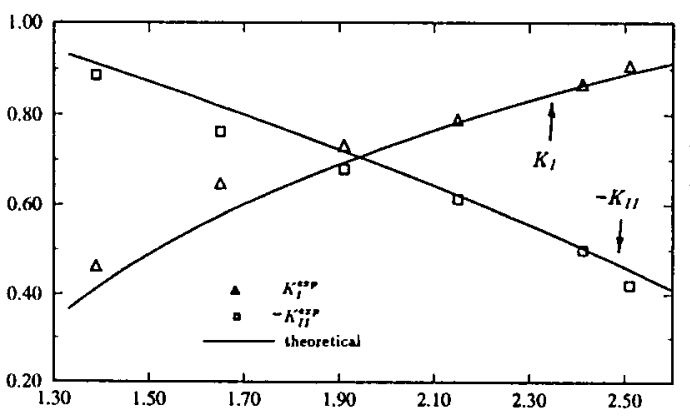

$(a / l)$

Fig. 8. Normalized mode-I and mode-II stress intensity factors vs. $(a / l)$ for homogeneous specimen.

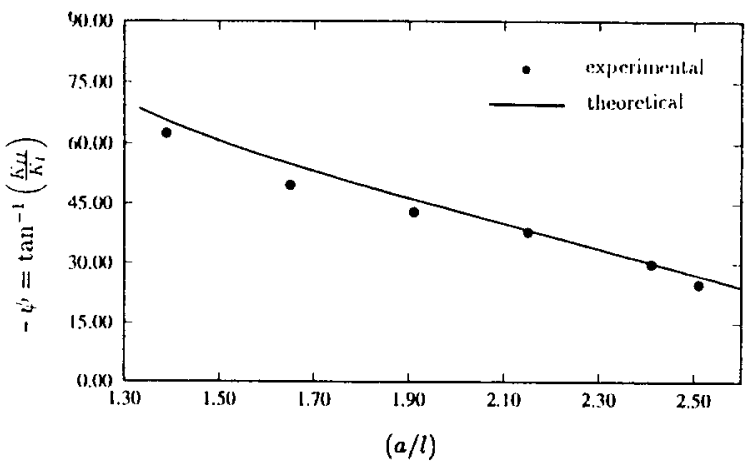

Fig. 9. Mode mixity vs. $(a / l)$ for homogeneous specimen.

ments. A fairly wide range of mode $\operatorname{mixities}\left(\psi \approx 20^{\circ}-65^{\circ}\right)$ are obtained in the range of $(a / l)$ used in the study. Table 1 summarizes all the results for the entire range of $(a / l)$ investigated. The load levels to which the stress intensity factors correspond are also listed. Two sets of results, namely the $K$-dominant case (when only the first term in the expansion field is used) and the asymptotic field case (when the optimum number of higher order terms are used), are presented in the table. The corresponding mode mixity parameters are also listed. The improvement in the experimental measurements upon incorporating higher order terms in the least-squares fit procedure is apparent. Interestingly, however, there is no appreciable change in the values of the mode mixity parameter for the $K$-dominant and the asymptotic field cases. Thus, it suggests that the $K$-dominant field itself may be sufficient in obtaining reasonably good estimates of the mode mixity parameter in this specimen geometry. Finally, the agreement between the experimental results and the corresponding beam theory predictions show the viability of CGS as an effective technique in performing direct mixed-mode crack tip measurements.

\section{Mixed-mode fracture at bimaterial interfaces}

\subsection{Bimaterial specimens and optical measurements}

Having observed a good agreement between experimental results and the corresponding theoretical values in homogeneous specimens, next we extend the use of the flexural specimen and the technique of CGS to study interface fracture mechanics. Based on the flexural geometry used in the study of homogeneous mixed-mode fracture, a single edge notched bimaterial fracture specimen is developed. The specimen geometry and the loading configuration used are shown in Fig. 10. The specimens are made from equal thickness $(B=9 \mathrm{~mm})$ sheets of PMMA $\left(E_{1}=3300 \mathrm{MPa}, v_{1}=0.35\right)$ and Aluminum 6061-T6 $\left(E_{2}=21 E_{1}, v_{2}=0.3\right)$. The height ratio $\xi$ $(=h / H)$ of the specimen is 0.74 such that the flexural rigidity of the upper and lower arms of the beam are the same. The span of the beam is $L=330 \mathrm{~mm}$ and the height $H=100 \mathrm{~mm}$. The Aluminum portion has a pre-cut notch of width $0.5 \mathrm{~mm}$ on its bond face. A hole of $6.25 \mathrm{~mm}$ is drilled in the Aluminum part of the specimen for applying the load $P$ as shown in Fig. 10. The specimen has a pre-cut slot on its notched edge for the purpose of housing a pin of $6.25 \mathrm{~mm}$ diameter. The pin provides reaction $P_{1}$ during loading. The bond face of the Aluminum piece is sand-blasted using approximately $20-50 \mu \mathrm{m}$ particles prior to bonding. The two materials are bonded to each other using a bonding agent (Weldon-10, manufactured by IPS Corporation, 


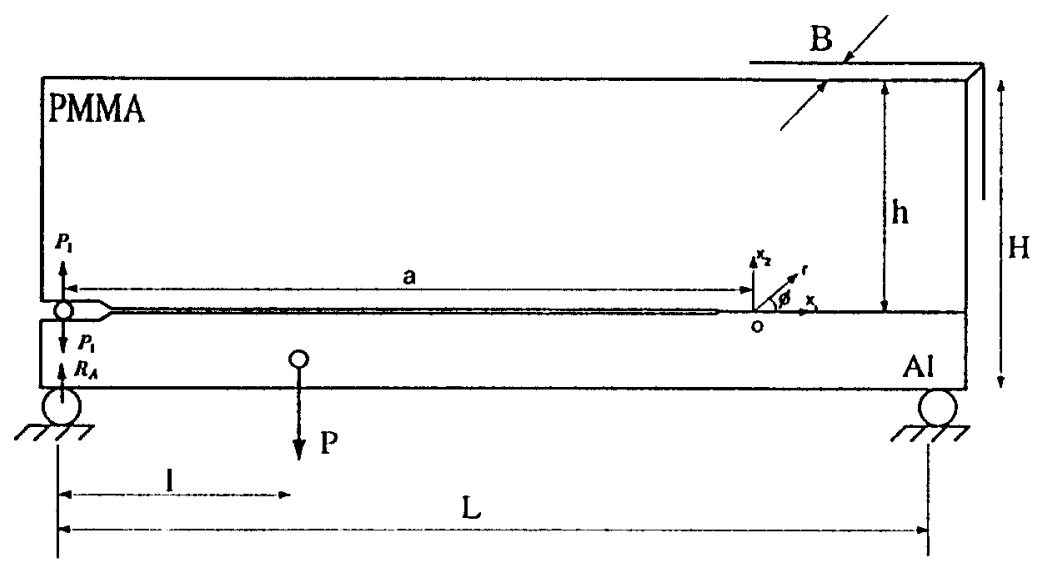

Fig. 10. Transversely cracked bimaterial specimen.

Gardena, CA) which consists of a methyl methacrylate monomer and a catalyst. The two components are mixed in the ratio $100: 13$ by weight. The bonding procedure used is similar to the one described in [19] and as recommended by the manufacturer. The stiffness characteristics of the bond material are similar to PMMA. During bonding, a teflon tape is used to create a 'crack-like' discontinuity along the bond. The bond is cured at room temperature for 24 hours and the bond thickness is observed to be approximately $50-100 \mu \mathrm{m}$. The interface is examined optically in the CGS set-up for residual stresses before using in the experiments. Absence of initial fringes, within the sensitivity limit of the technique, suggests a relatively residual stress-free interface.

Six different crack length $(a)$ to loading distance $(l)$ ratios, namely $(a / l)=1.45,1.68,1.98$, 2.21, 2.48 and 2.81, are studied. Transmission CGS is used in the investigation on bimaterial specimens. Hence, the experimental results come only from the PMMA half of the bimaterial specimen. A collimated laser beam of diameter $50 \mathrm{~mm}$ is incident on the region surrounding the crack tip. The pitch of the line gratings $p$ is $0.025 \mathrm{~mm}$ and the separation distance $\Delta$ is $39 \mathrm{~mm}$. The resulting interference fringe patterns are photographed at the image plane in real time. The load levels corresponding to the different patterns are measured using a load cell. For each specimen, the load corresponding to crack initiation at the interface $\left(P_{c r}\right)$ is also recorded. The transmission CGS fringe patterns around the crack tip for various $(a / l)$ ratios, when the grating lines are perpendicular to the $x_{1}$-axis, are shown in Fig. 11 (a) (c). The sensitivity of the measurement is $6.4 \times 10^{-4}$ radians per fringe.

\subsection{Interface crack tip fields}

Considering the material above the interface $(0<\phi<\pi)$, and following Rice, Suo and Wang [25], we can write

$$
\sigma_{11}+\sigma_{22}=\frac{2 e^{\varepsilon(\phi-\pi)}}{\sqrt{2 \pi r} \cosh (\pi \varepsilon)}\left[\left\{\operatorname{Re}\left(K a^{i \varepsilon}\right)\right\} \cos \left(\frac{1}{2} \phi+\varepsilon \ln \frac{r}{a}\right)-\left\{\operatorname{Im}\left(K a^{i \varepsilon}\right)\right\} \sin \left(\frac{1}{2} \phi+\varepsilon \ln \frac{r}{a}\right)\right]
$$


where $K=K_{1}+i K_{2}$ is the complex stress intensity factor. Here $\varepsilon$ is the stiffness mismatch parameter given by

$$
\varepsilon=\frac{1}{2 \pi} \ln \frac{\mu_{1}+\mu_{2} \kappa_{1}}{\mu_{2}+\mu_{1} \kappa_{2}}
$$

where $\kappa_{\alpha}=\left(3-v_{\alpha}\right) /\left(1+v_{\alpha}\right)$ for plane stress and $\mu$ represents shear modulus. The bimaterial phase angle $\psi^{b m}$, using crack length $a$ as the length scale, is defined as

$$
\psi^{b m}(a)=\tan ^{-1} \frac{\operatorname{Im}\left(K a^{i \varepsilon}\right)}{\operatorname{Re}\left(K a^{i \varepsilon}\right)} .
$$

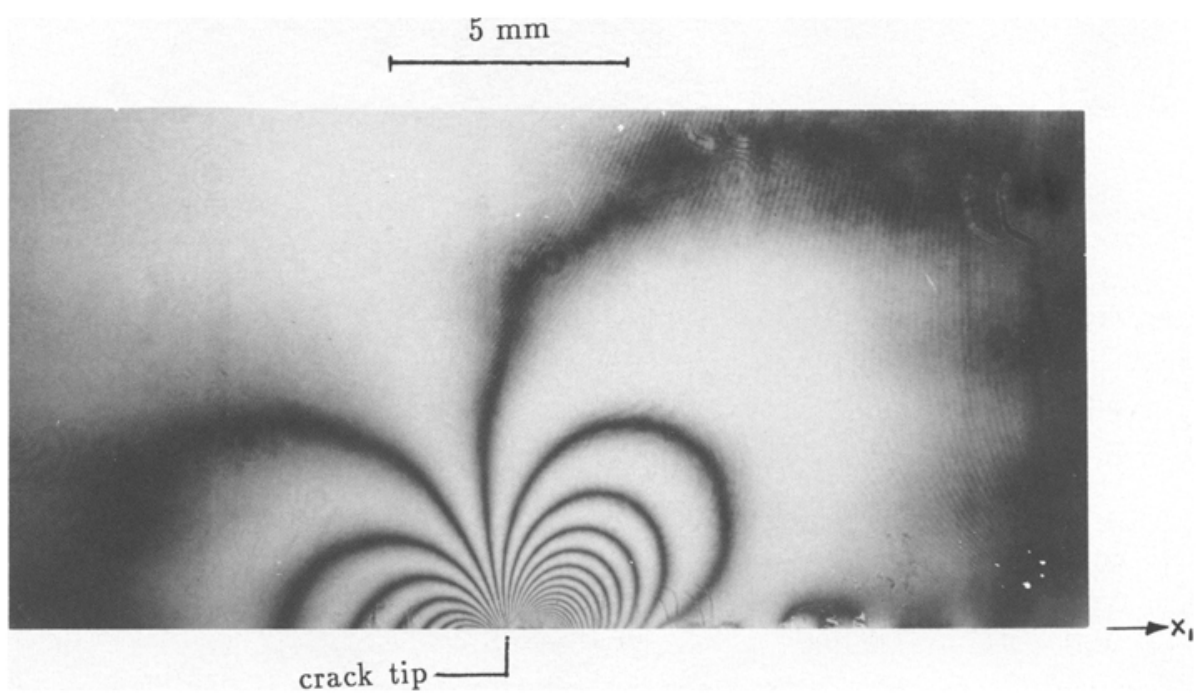

Fig. 11(a). Transmission CGS fringes representing contours of $\partial\left(\sigma_{11}+\sigma_{22}\right) / \partial x_{1}$ for the bimaterial specimen with $(a / l)=1.45$.

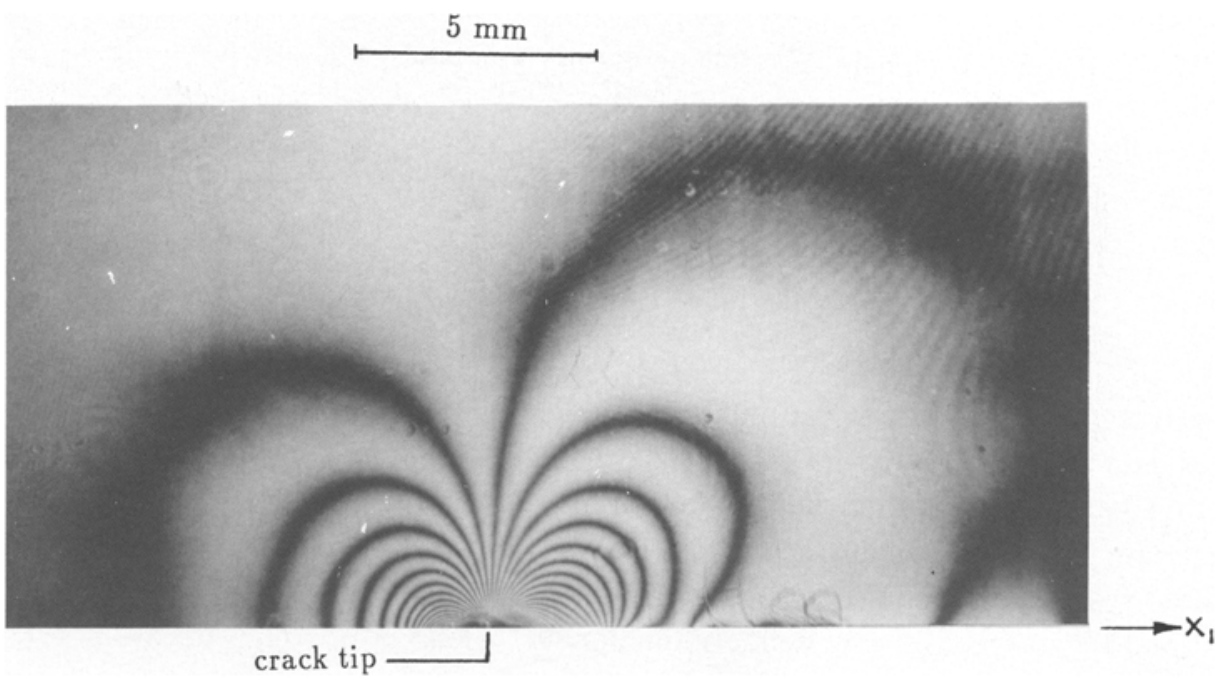

Fig. 11(b). Transmission CGS fringes representing contours of $\partial\left(\sigma_{11}+\sigma_{22}\right) / \partial x_{1}$ for the bimaterial specimen with $(a / l)=1.98$. 


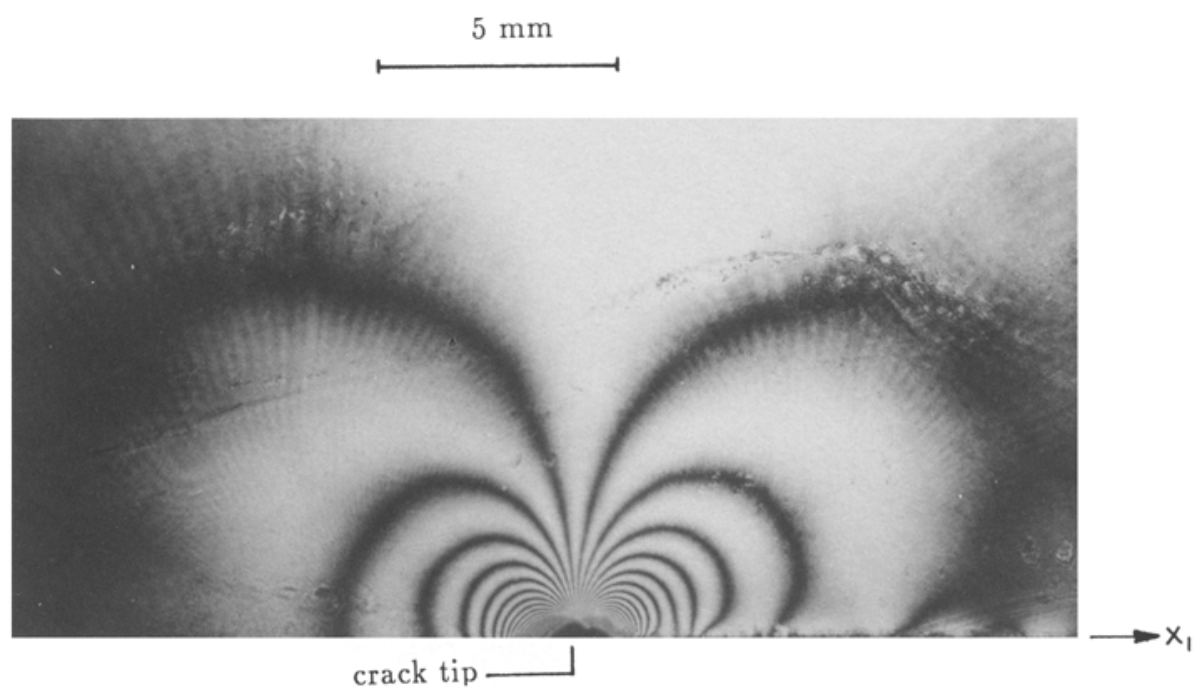

Fig. 11(c). Transmission CGS fringes representing contours of $\partial\left(\sigma_{11}+\sigma_{22}\right) / \partial x_{1}$ for the bimaterial specimen with $(a / l)=2.48$.

Also, when dealing with different crack lengths ( $\hat{a})$ (or other length scales), the phase angles are related by

$$
\hat{\psi}^{b m}(\hat{a})=\psi^{b m}(a)+\varepsilon \ln (\hat{a} / a) .
$$

Transmission CGS provides interference patterns that represent the deformation $c B\left(\partial\left(\sigma_{11}+\right.\right.$ $\left.\left.\sigma_{22}\right) / \partial x_{\alpha}\right)$. Hence, we get the relationship between the interface crack tip deformations and the interference patterns

$$
\begin{aligned}
c B \frac{\partial\left(\sigma_{11}+\sigma_{22}\right)}{\partial x_{1}}= & \frac{c B r^{-3 / 2} e^{\varepsilon(\phi-\pi)}}{\sqrt{2 \pi} \cosh (\pi \varepsilon)}\left[-\left\{\operatorname{Re}\left(K a^{i \varepsilon}\right)\right\} \cos \left(\frac{3 \phi}{2}+\varepsilon \ln \frac{r}{a}\right)\right. \\
& -2 \varepsilon\left\{\operatorname{Re}\left(K a^{i \varepsilon}\right)\right\} \sin \left(\frac{3 \phi}{2}+\varepsilon \ln \frac{r}{a}\right)+\left\{\operatorname{Im}\left(K a^{i \varepsilon}\right)\right\} \sin \left(\frac{3 \phi}{2}+\varepsilon \ln \frac{r}{a}\right) \\
& \left.-2 \varepsilon\left\{\operatorname{Im}\left(K a^{i \varepsilon}\right)\right\} \cos \left(\frac{3 \phi}{2}+\varepsilon \ln \frac{r}{a}\right)\right], \\
= & \frac{n_{1} p,}{\Delta}, \quad n_{1}=0, \pm 1, \pm 2 \ldots
\end{aligned}
$$

To extract the values of the stress intensity factors from the fringe patterns, we again use a leastsquares data analysis. The fringe patterns are digitized to obtain fringe location $(r, \phi)$ and fringe order $\left(n_{1}\right)$ data in the near tip region all around the crack tip. Recognizing the existence of a region of dominant 3-D deformations near the crack tip [26], only the data in the region $(r / B \leqslant 0.5$, $\left.-150^{\circ} \leqslant \phi \leqslant 45^{\circ}\right)$ near the crack tip are used in the analysis. Denoting the right hand sides of (14) by $Y^{b m}$ and $F^{b m}$, respectively, we define a function $\Phi^{b m}\left(\left\{\operatorname{Re}\left(K a^{i \varepsilon}\right)\right\}=Q_{1},\left\{\operatorname{Im}\left(K a^{i \varepsilon}\right)\right\}=Q_{2} ; r, \phi\right)$ as

$$
\Phi^{b m}=\sum_{i=1}^{M}\left[Y_{i}^{b m}-F_{i}^{b m}\right]^{2},
$$




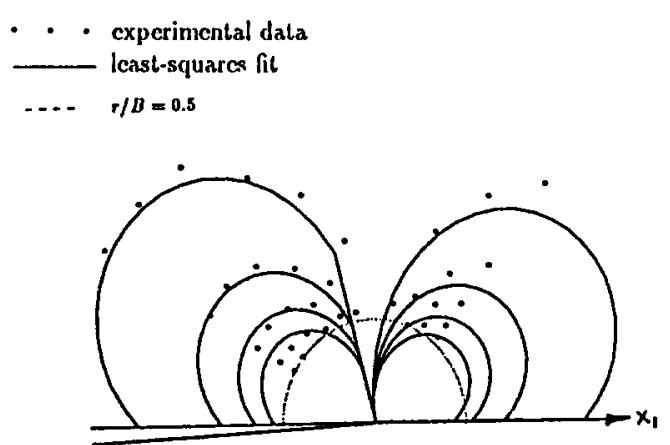

Fig. 12. Least-squares fit and data points for the case of $(a / l)=2.48$.

where $M$ is the total number of data points used in the analysis. In the curve fitting procedure, we minimize $\Phi^{b m}$ with respect to $Q_{1}$ and $Q_{2}$. The values of $Q_{1}$ and $Q_{2}$ obtained from the least squares analysis of the data are denoted by $Q_{1}^{\exp }$ and $Q_{2}^{\exp }$. Figure 12 shows a typical least squares fit for an $(a / l)$ ratio of 2.48 . The semicircle in the figure represents $(r / B)=0.5$. It should be pointed out here that the analysis is based on only the dominant term in the series expansion of the bimaterial interface crack tip. However, as observed in the homogeneous experiments (Table 1), the phase angle measurement is relatively insensitive to higher order terms for this specimen geometry and thus the phase angle measurements are reasonably accurate estimates. From the complex stress intensity factor, the energy release rate is calculated using

$$
G^{\exp }=\frac{1}{2}\left(\frac{1}{E_{1}}+\frac{1}{E_{2}}\right) \frac{K \bar{K}}{\cosh ^{2}(\pi \varepsilon)},
$$

where $\bar{K}$ is the complex conjugate of $K$, and $E_{1}$ and $E_{2}$ are the Young's moduli of the two materials. Here, $K \bar{K}=Q_{1}^{2}+Q_{2}^{2}$.

\subsection{Beam analysis for bimaterial specimens}

The experimental energy release rate $\left(G^{\exp }\right)$, for each $(a / l)$ ratio, is compared with its beam theory counterpart $\left(G^{b t}\right)$. Using an approach similar to the one mentioned in Section 3, the theoretical energy release rate for the bimaterial specimen can be shown to be

$$
G^{b t}=\frac{1}{2 B}\left[\frac{M_{1}^{2}}{E_{1} I_{1}}+\frac{M_{2}^{2}}{E_{2} I_{2}}-\frac{\left(M_{1}+M_{2}\right)^{2}}{E_{1} I_{0}}\right],
$$

where $M_{1}$ and $M_{2}$ are the crack tip moments in the upper and lower arms of the beam, $I_{1}, I_{2}$ and $I_{0}$ are the respective moments of inertia of the cross-sections of the upper arm, the lower arm and the transformed uncracked portion, $E_{1}$ is the Young's modulus of the material constituting the upper beam and $B$ is the out-of-plane thickness of the specimen. And the expressions of $M_{1}, \mathrm{M}_{2}$ and $I_{0}$ are

$$
\begin{aligned}
& M_{1}=P_{1} a, \\
& M_{2}=P\left(1-\frac{a}{L}\right) l-P_{1} a,
\end{aligned}
$$




$$
\begin{aligned}
& I_{0}=I_{1}+B h\left(H-\frac{1}{2} h-Z\right)^{2}+I_{2}+e B(H-h)\left(Z-\frac{1}{2}(H-h)\right)^{2}, \\
& Z=\frac{1}{H}\left[h\left(H-\frac{1}{2} h\right)+\frac{1}{2}(H-h)^{2}\right], \\
& e=\frac{E_{2}}{E_{1}}
\end{aligned}
$$

where $P_{1}$ is the force between the two arms given in Section 3.4 and is valid when $E_{1} I_{1}=E_{2} I_{2}$.

It should be noted here that, mode-partitioning using moment decomposition technique is not feasible in this instance because $\xi \neq 0.5$ as in the case of homogeneous specimen. Alternative analyses such as the interaction integral method proposed in [12] are necessary to independently measure the phase of $\left(K a^{i \varepsilon}\right)$ and will not be pursued here any further.

\subsection{Results for bimaterial specimens}

The results for the bimaterial specimens are shown in Figs. 13-15 and Table 2. The comparison between the measured and the predicted energy release rates for the different $(a / l)$ ratios considered is shown in Fig. 13. The solid line in the figure represents $G^{\exp } / G^{b t}=1$ and the circles represent the experimental data. Experimestal measurements are within $\pm 10 \%$ of the beam theory values reflecting a fairly good agreement between them. Figure 14 shows the plot of the mixity parameter, $\psi^{b m}=\tan ^{-1}\left(\left\{\operatorname{Im}\left(K a^{i \varepsilon}\right)\right\} /\left\{\operatorname{Re}\left(K a^{i \varepsilon}\right)\right\}\right)$ vs. $(a / l)$. A fairly wide range of mode mixities with crack length as the scaling parameter $\left(\psi^{b m} \approx 10^{\circ}-50^{\circ}\right)$ are observed in the range of $(a / l)$ ratios tested. Also, using the load at crack initiation $P_{c r}$, critical energy release rate $G_{c r}$, is calculated. The variation of crack initiation toughness is plotted against experimentally measured phase angle $\left(G_{c r}\right.$ vs. $\left.\hat{\psi}^{b m}\right)$ in Fig. 15. Here, phase angles are calculated based upon a scaling parameter of $\hat{a}=0.145 \mathrm{~m}$. As one would anticipate, significantly higher values of $G_{c r}$ are seen when the crack is subjected to predominantly shear loading when compared with the ones for predominantly tensile loading. This is qualitatively similar to the steady state toughness values presented for glass-epoxy interface in [15] wherein bond-tangential loadings are shown

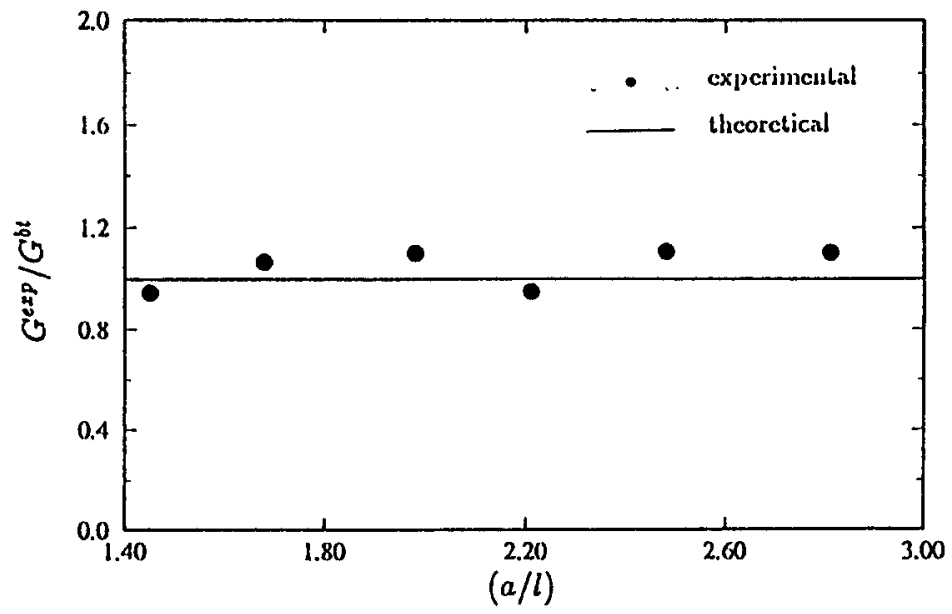

Fig. 13. Comparison of experimental and beam theory energy release rates for bimaterial specimens. 


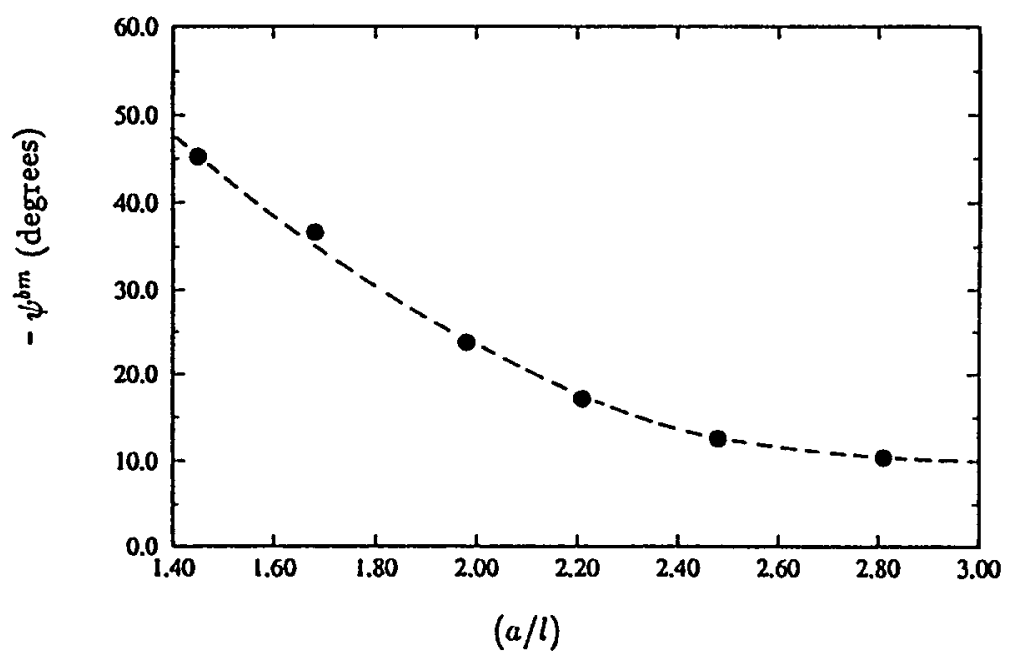

Fig. 14. Plot of mode mixity vs. $(a / l)$.

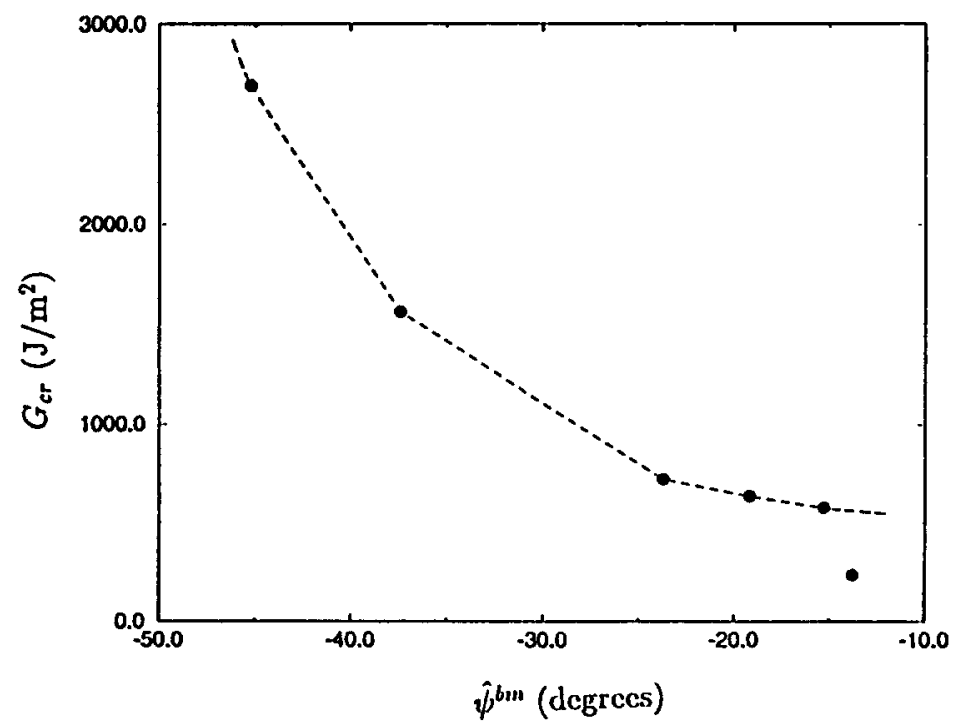

Fig. 15. Plot of crack initiation toughness vs. mode mixity for PMMA-Al interface.

Table 2. Comparison of experimental and beam theory results for bimaterial specimens

\begin{tabular}{llllllllll}
\hline$(a / l)$ & $P$ & $G^{b t}$ & $Q_{1}=\operatorname{Re}\left[K a^{i e}\right]$ & $Q_{2}=\operatorname{Im}\left[K a^{i \varepsilon}\right]$ & $G^{\exp }$ & $\psi^{b m}(a)$ & $\hat{\psi}^{b m}(\hat{a})$ & $P_{c r}$ & $G_{c r}$ \\
\hline 1.45 & 3172 & 823 & 1.62 & -1.63 & 779 & -45.2 & -45.2 & 5736 & 2693 \\
1.68 & 3164 & 770 & 1.90 & -1.41 & 821 & -36.6 & -37.4 & 4500 & 1557 \\
1.98 & 3130 & 695 & 2.09 & -0.92 & 764 & -23.7 & -25.3 & 3188 & 721 \\
2.21 & 3003 & 594 & 1.87 & -0.58 & 565 & -17.1 & -19.2 & 3103 & 634 \\
2.48 & 2976 & 548 & 1.98 & -0.44 & 607 & -12.6 & -15.3 & 3051 & 576 \\
2.81 & 1739 & 176 & 1.13 & -0.21 & 194 & -10.4 & -13.8 & 1947 & 221 \\
\hline
\end{tabular}

$Q=K a^{i \varepsilon}$ values are in $\mathrm{MPa}(\mathrm{m})^{1 / 2+i \varepsilon}, P$ in Newtons, $G$ in $J / \mathrm{m}^{2}$, and $\psi$ in degrees.

$(*)^{b t}=$ values based on beam theory, $(*)^{b m}$ are values for bimaterial specimens, $\hat{a}=0.145 \mathrm{~m}$ 
to produce significantly higher (approximately eight times) toughness when compared to bond-normal loading. In view of high $G_{c r}$ values for larger phase angles observed in this study, one may anticipate crack branching into PMMA. Surprisingly, branching of the crack into PMMA seems to be absent at initiation. However, fractured surfaces for shorter crack lengths often showed small chunks of broken PMMA or the bond material on broken Aluminum surface suggesting a rather complex fracture process. Other contributing factors to such a behavior could be (i) finite crack tip radius due to the thickness of the bond, and (ii) the intentionally introduced surface roughness (by sand blasting the Aluminum half) prior to bonding in order to enhance bond strength. The surface roughness effects such as interlocking between crack face asperities considered by Evans and Hutchinson [27] could possibly be significant in this case. Also, in Fig. 15, the measured $G_{c r}$ values for smaller phase angles are comparable to the quasi-static values tabulated in [19] for different crack length to beam height ratios obtained using symmetric three point bend bimaterial fracture specimens made of PMMA-Aluminum. A summary of results from experiments and beam theory for different $(a / l)$ ratios is presented in Table 2. Small differences between $\psi^{b m}$ and $\hat{\psi}^{b m}$ should be noted.

\section{Conclusions}

An investigation of mixed-mode fracture in homogeneous and bimaterial solids under elastostatic conditions is carried out. A modified edge notched flexural specimen is proposed to investigate bimaterial interface fracture. The capability of the specimen in providing a wide range of mode mixities is first established in the homogeneous case and then tested in the bimaterial case. A full field optical technique, CGS, is used to directly map crack tip deformations and measure crack tip parameters.

In the homogeneous case, Williams' expansion field is used to analyze interference patterns in the experimental determination of crack tip parameters. The experimental results, stress intensity factors $\left(K_{1}\right.$ and $\left.K_{\mathrm{II}}\right)$ and mode mixity $(\psi)$, are found to agree well with the theoretical predictions. A wide range of mode mixities $\left(\psi(a)\right.$ varies approximately from $\left.20^{\circ}-65^{\circ}\right)$ is observed in experiments with homogeneous specimens.

In the bimaterial specimens, however, owing to the elastic stiffness mismatch across the interface, the crack tip fields are intrinsically mixed-mode. The combined effect of the loading geometry and stiffness mismatch have been analyzed by interpreting CGS fringes using two-dimensional interface crack tip fields. In this study, the analysis of the fringes is, however, restricted to the use of the dominant singular term in the 2-D interface crack tip field. Experimental results for energy release rate $\left(G^{\exp }\right)$ using the proposed bimaterial fracture specimens agree to within $\pm 10 \%$ of their theoretical counterparts. A fairly wide range of mode mixities $\left(\psi^{b m}\right.$ varies from $\left.10^{\circ}-50^{\circ}\right)$ have been observed. The dependence of interfacial crack initiation toughness $\left(G_{c r}\right)$ on the crack tip mode mixity parameter is shown experimentally for the PMMA-Al bimaterial system. The proposed single crack tip bimaterial specimen has advantages over two crack tip configurations. Advantages are that it greatly reduces loading symmetry restrictions that are often encountered in dual crack tip configurations such as a bimaterial Brazilian disk or a transversely cracked four point bend specimen. A somewhat gradual variation of mode mixity with the parameter $(a / l)$ seen in this investigation could also be advantageous in fracture toughness testing. 


\section{Acknowledgments}

The research is supported by NSF Research Initiation Award MSS9109731 to HVT. Equipment assistance by Auburn University through 1991 Research Grant-in-Aid is also gratefully acknowledged.

\section{References}

1. M.L. Williams, Bulletin of the Seismological Society of America 49(2) (1959) 199-203.

2. G.C. Sih and J.R. Rice, Journal of Applied Mechanics 31 (1964) 477-482.

3. G.C. Sih and J.R. Rice, Journal of Applied Mechanics 32 (1965) 418-423.

4. F. Erdogan, Journal of Applied Mechanics 32 (1965) 403-410.

5. A.H. England, Journal of Applied Mechanics 32 (1965) 400-402.

6. M. Comninou, Journal of Applied Mechanics 44 (1977) 631-636.

7. J.K. Knowles and R.L. Sternberg, Journal of Elasticity 13 (1983) 257-293.

8. C.F. Shih and R. Asaro, Journal of Applied Mechanics 55 (1988) 299-316.

9. N. Aravas and S.M. Sharma, Journal of the Mechanics and Physics of Solids 39(3), (1991) 311-344.

10. J.R. Rice, Journal of Applied Mechanics 55 (1988) 98-103.

11. J.W. Hutchinson, M.E. Mear and J.R. Rice, Journal of Applied Mechanics 54 (1987) 828-832.

12. N.P. O'Dowd, C.F. Shih and M.G. Stout, International Journal of Solids and Structures 29(5), (1992) 571-589.

13. J.W. Hutchinson and Z. Suo, Advances in Applied Mechanics 29 (1992) 63-191.

14. K.M. Liechti and W.G. Knauss, Experimental Mechanics 22 (1982) 383-391.

15. K.M. Liechti and Y.S. Chai, EMRL Report No. 89/4, The University of Texas at Austin (1990).

16. F.P. Chiang, L. Hua and X.T. Yan in Advances of Fracture Research, Proceedings of ICF-7, 4 (1989) 3063-3072.

17. H.C. Cao and A.G. Evans, Mechanics of Materials (1989) 295-301.

18. P.G. Charalambides, J. Lund, A.G. Evans and R.M. McMeeking, Journal of Applied Mechanics 56 (1989) $77-82$.

19. H.V. Tippur and A.J. Rosakis, Experimental Mechanics 31(3), (1991) 243-252.

20. H.V. Tippur, S. Krishnaswamy and A.J. Rosakis, International Journal of Fracture 52 (1991) 91-117.

21. M.L. Williams, Journal of Applied Mechanics 24 (1959) 109-112.

22. A.J. Rosakis and K. Ravi-Chandar, International Journal of Solids and Structures 22(2), (1986) 121-134.

23. S. Ramaswamy, M.S. thesis, Auburn University, Auburn, Alabama (1992).

24. J.G. Williams, International Journal of Fracture 36 (1988) 101-119.

25. J.R. Rice, Z. Suo and J.S. Wang, Metallurgica Proceedings Series, 4 (1990) 269-294.

26. T. Nakamura, Journal of Applied Mechanics 58 (1991) 939-946.

27. A.G. Evans, and J.W. Hutchinson, Acta Metallurgica 37 (1989) 909-916. 